\title{
Österreichische Arbeitsgemeinschaft für Neuropathologie. Wissenschaftliche Jahrestagung am 9. April 1978 in Innsbruck
}

\section{Programm}

\section{Technische und methodische Fortschritte in der Neuropathologie}

1. W. Schlote (Tübingen): Ortsbezogene Elementbestimmung im Schnittpräparat durch Röntgenstrahlenanalyse - Bedeutung für die Neuropathologie

2. J.Mai (Düsseldorf): Ein automatisiertes System zur quantitativen Analyse von Autoradiogrammen

3. P.Schubert, P.Mehraein, G.W.Kreutzberg (München) : Mögliche Anwendung der Einzelzell-Injektionstechnik kombiniert mit Autoradiographie in der Neuropathologie

4. G. Brandt, P.Thierauf, K. Metze (Erlangen): Diagnostik postmortaler Elektrolytstörungen im Hirngewebe mit der Gewebsmineralanalyse

5. G.F. Walter, H.Höfler, O. Wawschinek (Graz) : ,MDCM“- Methode zum histochemischen Kupfernachweis bei hepatolentikulärer Degeneration (Morbus Wilson)

6. H. H. Goebel, B. Sakmann, W. Gerlach (Göttingen): Ultrastrukturelle Darstellung muskulärer Acetylcholinrezeptoren

7. K. Jellinger, P. Riederer (Wien): Die Bedeutung biogener Amine beim Hirninfarkt

8. P.Mehraein, H.Holländer, H.W. Dietl (München): Einsatzmöglichkeit eines Flächen- und Koordinatenregristrierenden Mikroskops bei quantitativen Untersuchungen in der Neuropathologie

9. H.P.Schmitt (Heidelberg): Probleme der Querschnittsvermessung an Skelettmuskelfasern. Vergleich der Dignität verschiedener Methoden und Parameter

10. F. D. Goebel, H. H. Goebel (Göttingen): Quantifizierung der Basalmembrandicke muskulärer Kapillaren mit Hilfe des Elektronenmikroskops
11. R.Schmidt, H.-P.Dienes, S.Kunowski, H.Herbst (Berlin): Licht-, elektronen- und fluoreszenzmikroskopische Studie zur Entwicklung des vegetativen intrapulmonalen Nervensystems

\section{Freie Themen}

12. H.Budka, F. Seitelberger (Wien): Konnatale leukodystrophische Prozesse

13. H.Bernheimer, H.Blinzinger, K.Jellinger, B.Molzer (Wien u. München): Adulte metachromatische Leukodystrophie: licht-, elektronenoptische und neurochemische Befunde

14. J.W.Boellaard (Tübingen): Über einen Fall von atypischer Alzheimerscher Erkrankung mit ${ }^{1 / 2}$ jähriger Verlaufsdauer

15. H.Gross, H.Pfolz (Wien): Ungewöhnliche Form einer präsenilen Demenz

16. M.Simanyi, (Wien): Organisches Psychosyndrom und neuropathologische Befunde bei Epilepsie

17. G. Lassmann, H.Lassmann, W.Jurecka, H.Matras, G. Watzek (Wien): Plexiformes Neurofibrom mit Tastkörperchenstruktur

18. K. Bisse, O.Stochdorph (München): Über seltene Varianten von Hypophysentumoren

19. P. Pilz, M. Brenneis (Salzburg): Intrakranielle Thrombophlebitis und Phlebothrombose

20. H.Auff, H.Budka (Wien): Meningeale Karzinomatosen

21. G.Lassmann (Wien): Neurohistologische Befunde bei Analatresie 